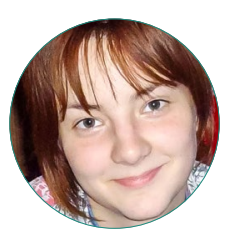

С.В. Докука

\title{
ИСПОЛЬЗОВАНИЕ СТОХАСТИЧЕСКИХ АКТОРНО-ОРИЕНТИРОВАННЫХ МОДЕЛЕЙ ДЛЯ АНАЛИЗА КОЭВОЛЮЦИИ СЕТЕЙ И ПОВЕДЕНИЯ
}

\section{Правильная ссылка на статью:}

Докука С.В. Использование стохастических акторно-ориентированных моделей для анализа коэволюции сетей и поведения // Мониторинг общественного мнения: экономические и социальные перемены. 2021. № 2. С. 273-285. https://doi.org/10.14515/ monitoring.2021.2.1806.

\section{For citation:}

Dokuka S. V. (2021) Stochastic Actor-Oriented Models in the Analysis of Co-Evolution of Networks and Behavior. Monitoring of Public Opinion: Economic and Social Changes. No. 2. P. 273-285. https://doi.org/10.14515/monitoring.2021.2.1806. (In Russ.) 
ИСПОЛЬЗОВАНИЕ СТОХАСТИЧЕСКИХ АКТОРНО-ОРИЕНТИРОВАННЫХ МОДЕЛЕЙ ДЛЯ АНАЛИЗА КОЭВОЛЮЦИИ СЕТЕЙ И ПОВЕДЕНИЯ

ДОКУКА София Владимировна - кандидат социологических наук, научный сотрудник Лаборатории вычислительных социальных наук Института образования, Национальный исследовательский университет "Высшая школа экономики", Москва, Россия

E-MAIL: sdokuka@hse.ru

https://orcid.org/0000-0002-0847-5129

Аннотация. Поведение человека зависит от многих факторов, в том числе и от социального окружения. В течение последних десятилетий активно разрабатывались модели, оценивающие влияние окружения на индивида. В настоящей статье описывается стохастическое акторно-ориентированное моделирование (САОМ), позволяющее тестировать гипотезы, касающиеся структурной эволюции социальных сетей, а также детально анализировать коэволюцию сетей и поведения. В работе описаны теоретико-методологические основания САОМ, приведены требования к дизайну исследований и процедурам сбора данных, рассмотрены спецификация модели и пример коэволюции сети и академических достижений учащихся, отмечены ограничения метода, а также предложены перспективные направления для дальнейшего изучения. Мы указываем, что анализ с использованием САОМ предпочтителен для небольших социальных сетей с низкой ротацией, в частности организационных подразделений и учебных групп. В статье отмечается, что существующие работы рассматривают преимущественно образова-
STOCHASTIC ACTOR-ORIENTED MODELS IN THE ANALYSIS OF CO-EVOLUTION OF NETWORKS AND BEHAVIOR

Sofia V. DOKUKA 1 -Cand. Sci. (Soc.), Researcher at the Laboratory for Computational Social Science, Institute of Education E-MAIL:sdokuka@hse.ru https://orcid.org/0000-0002-0847-5129

\footnotetext{
1 National Research University Higher School of Economics, Moscow, Russia
}

Abstract. Human behavior depends on many factors including the social environment. Over the past decades, scholars have actively developed models that evaluate the impact of the environment on the individual. This article describes stochastic actor-oriented modeling (SAOM) which allows to test hypotheses about the structural evolution of social networks, as well as to analyze the co-evolution of networks and behavior in detail. The authors present theoretical and methodological foundations of SAOM; specify requirements for research design and data collection; consider the specification of the model and an example of co-evolution of the network and academic achievement of students; point out limitations of the method, and suggest promising avenues for further research. The article concludes that using SAOM is preferable for small social networks with low rotation, in particular, for organizational units and study groups. The authors argue that the existing studies mainly consider the educational context and social networks of young people that does not allow to draw conclusions about the evolution of social ties in communities of a different type and other age 
тельный контекст и социальные сети молодых людей, что не позволяет сделать выводы о механизмах эволюции социальных связей в общностях иного типа и для представителей других возрастных групп. Также отмечается, что подавляющее большинство исследований реализовано на данных о западных демократических обществах, что свидетельствует о необходимости изучения разнообразных выборок.

Ключевые слова: анализ социальных сетей, стохастические акторно-ориентированные модели, социология образования, академические достижения, эффекты сообучения

Благодарность. Исследование осуществлено в рамках Программы фундаментальных исследований НИУ ВШЭ. groups. In addition, most of the research is based on data collected in Western democracies which indicates the need to investigate a variety of samples.

Keywords: social network analysis, peer effect, sociology of education, academic achievements, stochastic actor-oriented models

Acknowledgments. The research was carried out within the HSE Program for Fundamental Research.

\section{Введение}

На индивидуальное поведение оказывает влияние большое количество факторов, и особое место среди них занимает социальное окружение. Оценкой влияния окружающих на индивидуальное поведение социологи заинтересовались в 1930-х годах, и одним из первопроходцев данного направления стал Якоб Морено [Prell, 2012; Wasserman, Faust, 1994], изучавший взаимосвязь между благополучием человека (далее - актора) и социальным окружением. Морено разработал социометрию - методический аппарат для сбора данных о социальных взаимоотношениях индивидов (далее - связей) внутри небольших групп.

Эти наработки стали базой для формирующегося анализа социальных сетей. В 1970-х годах начался рост интереса к этому направлению, сопровождавшийся формализацией сетевого аппарата на базе инструментария теории графов [Харари, 1973]. Анализ социальных сетей востребован для репрезентации большого числа социальных систем, однако его применение поставило исследователей перед новыми методологическими вызовами. Самый существенный вызов в том, что акторы социальной сети связаны и влияют на поведение друг друга. В свою очередь, ключевая предпосылка линейных методов - независимость наблюдений друг от друга. Соответственно, линейные методы (корреляции, регрессии) не могут быть использованы для оценки взаимосвязи между положением в социальной сети и индивидуальными характеристиками [Robins, 2013], что диктует необходимость разработки новых подходов [Докука, Валеева, 2015]. 
Одним из таких подходов стали р*-модели, или экспоненциальные модели случайных графов (Exponential Random Graph Models, ERGM). B ERGM наблюдаемая социальная сеть рассматривается как одна из возможных реализаций сети с заданными параметрами (число вершин, число ребер, склонность людей со схожими характеристиками быть связанными друг с другом) [Wasserman, Faust, 1994; Robins et al., 2007]. В ходе моделирования ставится задача выявить структурные и иные характеристики социальной системы, отличающие наблюдаемую сеть от случайных. К примеру, в исследовании [Валеева, Польдин, Юдкевич, 2013] показано, что студенты склонны формировать связи с одногруппниками, а также с учащимися одного пола. При этом академически результативные студенты склонны получать запросы о помощи, но не о дружбе. B работе [Grunspan et al., 2016] с использованием ERGM была проанализирована сеть взаимоотношений студентов-биологов. Выяснилось, что мужчин чаще указывают в качестве успешных студентов, при этом их популярность обусловлена не только академической успешностью. Число номинаций женщин в качестве успешных студентов объясняется через их академические достижения. Хотя ERGM позволяет одновременно рассматривать как связи, так и индивидуальные характеристики, в рамках этого метода невозможно сделать выводы о причинно-следственных связях и определить, существует ли влияние на поведение акторов со стороны социального окружения.

Для выявления каузальности в сетевых работах и ответов на более амбициозные исследовательские вопросы необходимы как иные типы данных (в частности, лонгитюдные), так и иной инструментарий. Для реализации этих задач Снайдерс с коллегами разработал стохастические акторно-ориентированные модели (далее-CAOM) [Snijders, Van de Bunt, Steglich, 2010; Ripley et al., 2020], которым и посвящена данная статья. Далее будут описаны основные теоретические предпосылки метода, методика дизайна исследования, требования к выборке, процедуры анализа с использованием САОМ на примере сети студентов, а также отмечены ограничения метода. В заключении обсуждаются перспективные направления в рамках данной тематики.

\section{Теоретические основания САОМ}

САОМ используют для изучения эволюции социальной сети (и поведения) на основе лонгитюдных данных. Принципиальное преимущество этих моделей возможность одновременной репрезентации сразу нескольких динамических сетевых процессов.

Важно отметить, что в рамках САОМ не рассматриваются предпосылки изначального возникновения связей. Анализируются именно процессы, лежащие в основе изменения сети (и поведения акторов) в период $t_{1} \rightarrow t_{2}$. Эти изменения рассматриваются как результат марковского процесса, при котором на состояние системы в момент времени $t_{2}$ влияет исключительно состояние системы в $t_{1}$.

САОМ опирается на ряд следующих предпосылок.

В качестве связей рассматриваются состояния, а не краткосрочные события. К состояниям можно отнести сеть дружбы, помощи, позитивных или негативных взаимоотношений, совместную работу. К событиям - телефонные звонки, пере- 
писку по электронной почте и в социальных медиа. При этом Снайдерс отмечает, что данные по событиям можно анализировать в агрегированном виде [Snijders, Van de Bunt, Steglich, 2010].

Акторы действуют в соответствии с предпосылкой о социальном индивидуализме. Они контролируют свои исходящие связи и принимают решение об изменении связи (или поведения, при условии моделирования коэволюции сетей и поведения), опираясь на информацию о структуре всей сети и поведении всех индивидов в ней. Таким образом, акторы информированы о поведении и связях всех индивидов группы. Как отмечают разработчики, это предположение не всегда реалистично, часто актор может быть осведомлен только о действиях своего непосредственного окружения и не имеет полных данных об остальных.

Макроизменения в структуре сети рассматриваются как последовательность микроизменений. В каждый момент времени актор может изменить либо поведение, либо связь. Результирующие макроизменения являются результатом последовательно происходящих микроизменений.

Для описания изменений в сети используется целевая функция. Как отмечает Снайдерс [Snijders, Van de Bunt, Steglich, 2010], она описывает «правила сетевого поведения" для акторов. Функция определяется как набор возможных состояний сети (и поведения) с точки зрения конкретного актора. По аналогии с обобщенными линейными моделями целевая функция $\left(f_{\mathrm{i}}\right)$ определяется как линейная комбинация эффектов:

$$
f_{i}(\beta, x)=\sum_{k} \beta_{k} s_{k i}(x)
$$

где $s_{k i}(x)$ - это аналитическая функция (также обозначаемая как эффект), описывающая сетевые тенденции, $\beta_{k}$ - статистический параметр, демонстрирующий статистическую значимость эффекта.

Вариации сетевых эффектов многочисленны. Мы остановимся на базовых эффектах, наиболее часто использующихся в САОМ-моделировании (см. табл. 1). По аналогии с другими регрессионными моделями САОМ позволяет формировать более сложные интеракционные эффекты [Block, Grund, 2014; Kretschmer, Leszczensky, Pink, 2018].

Таблица 1. Сетевые и поведенческие динамические эффекты

\begin{tabular}{|c|c|c|}
\hline Эффект & Визуализация & Интерпретация \\
\hline $\begin{array}{l}\text { Плотность } \\
\text { (outdegree) }\end{array}$ & & $\begin{array}{l}\text { Негативный эффект демонстрирует } \\
\text { отсутствие склонности акторов } \\
\text { к формированию связей. }\end{array}$ \\
\hline $\begin{array}{l}\text { Взаимность } \\
\text { (reciprocity) }\end{array}$ & & $\begin{array}{l}\text { Положительный эффект } \\
\text { обозначает склонность } \\
\text { к формированию } \\
\text { взаимных связей. }\end{array}$ \\
\hline
\end{tabular}




\begin{tabular}{|c|c|c|}
\hline Эффект & Визуализация & Интерпретация \\
\hline $\begin{array}{l}\text { Транзитивность } \\
\text { (transivitity) }\end{array}$ & & $\begin{array}{l}\text { Положительный эффект } \\
\text { свидетельствует о формировании } \\
\text { транзитивных связей (по принципу } \\
\text { “друг моего друга - мой друг»). }\end{array}$ \\
\hline $\begin{array}{l}\text { 3-циклы } \\
\text { (3-cycles) }\end{array}$ & & $\begin{array}{l}\text { Положительный эффект } \\
\text { 3-циклов обозначает тенденцию } \\
\text { к формированию замкнутых } \\
\text { циклических структур. }\end{array}$ \\
\hline $\begin{array}{l}\text { Популярность } \\
\text { (popularity) }\end{array}$ & & $\begin{array}{l}\text { Положительная популярность } \\
\text { свидетельствует о том, что ряд } \\
\text { акторов с течением времени } \\
\text { получает большее число } \\
\text { номинаций. }\end{array}$ \\
\hline $\begin{array}{l}\text { Активность } \\
\text { (activity) }\end{array}$ & & $\begin{array}{l}\text { Положительная активность } \\
\text { свидетельствует о том, что ряд } \\
\text { акторов с течением времени } \\
\text { указывает большее число друзей. }\end{array}$ \\
\hline $\begin{array}{l}\text { Социальная } \\
\text { селекция } \\
\text { (social selection) }\end{array}$ & & $\begin{array}{l}\text { Положительный эффект } \\
\text { социальной селекции } \\
\text { свидетельствует о тенденции } \\
\text { к формированию связей } \\
\text { между схожими акторами. }\end{array}$ \\
\hline $\begin{array}{l}\text { Социальное } \\
\text { влияние } \\
\text { (social influence) }\end{array}$ & & $\begin{array}{l}\text { Положительный эффект } \\
\text { социального влияния } \\
\text { свидетельствует о тенденции } \\
\text { к адаптации поведения } \\
\text { в паре связных акторов. }\end{array}$ \\
\hline
\end{tabular}

Оценки модели интерпретируются как коэффициенты логистической регрессии. Например, положительное и статистически значимое значение эффекта взаимности будет свидетельствовать о том, что в социальной сети участники стремятся к формированию взаимных связей [Snijders, Van de Bunt, Steglich, 2010].

\section{Дизайн исследования}

Использование САОМ предполагает специфический дизайн исследования. Одна из фундаментальных предпосылок модели (отмеченной в пункте «Теоретические основания САОМ") - предположение о полной информированности индивидов о структуре социальных связей и поведения. Несмотря на указание авторов, что это предположение часто не соответствует действительности, моделирование в САОМ больших систем (больше нескольких сотен вершин) не рекомендуется. 
Чаще всего исследования проводятся на данных о поведении учеников в классе [Rambaran et al., 2017; Palacios et al., 2019] или поведении сотрудников организации [Ellwardt, Steglich, Wittek, 2012]. Такие группы подразумевают низкую ротацию участников. Для реализации САОМ требуется лонгитюдный сбор неанонимных сетевых данных - в случае анонимизированных данных становится невозможным выстраивание полных сетей.

В ходе исследования обычно проводятся два-четыре опроса, в ходе которых фиксируются взаимоотношения между акторами, а также производится сбор данных об их индивидуальных характеристиках. Как и ERGM, CAOM требует максимально полной информации о структуре социальной сети. Разработчики рекомендуют сбор данных как минимум о 80 \% участников социальной группы, причем данных, касающихся как структуры сети, так и индивидуальных характеристик [Ripley et al., 2020]. При этом в последние годы растет число методов восполнения потерянных данных для подобных исследований [de la Haye et al., 2017; Zandberg, Huisman, 2019].

Важно, чтобы структура социальной сети изменялась со временем. Изменения оцениваются коэффициентом Жаккара [Jaccard, 1900], для САОМ он должен быть в интервале 0,3-0,7. В случае высоких или низких значений, обозначающих сильное сходство или сильное различие сети в моменты $t_{1}$ и $t_{2}$, соответственно, использование САОМ может быть затруднительным [Snijders, Van de Bunt, Steglich, 2010].

Моделирование с использованием САОМ проводится в статистической среде R с использованием пакета Rsiena [Ripley et al., 2020].

\section{Пример коэволюции социальной сети и академической успеваемости студентов}

Проиллюстрируем использование САОМ на примере работы, рассматривающей коэволюцию сети студентов и академической успеваемости учащихся первого курса экономического факультета высокоселективного российского университета [Dokuka, Valeeva, Yudkevich, 2020]. CAOM часто используется для изучения сетей в образовании [Lomi et al., 2011; Flashman, 2012; DeLay et al., 2018; Brouwer et al., 2018]. Это связано с относительной доступностью данных, а также устойчивостью социальных групп.

В работе [Dokuka, Valeeva, Yudkevich, 2020] были проанализированы сети дружбы и помощи и их связь с академической успеваемостью. Данные были собраны трижды: в октябре 2013 г. (начало обучения в университете), в феврале 2014 г. (после сессии), в июне 2014 г. (во время последней сессии года). Учащихся опросили об их связях дружбы (“С кем Вы проводите много времени вместе?») и связях помощи («К кому Вы обращаетесь за помощью в учебе?»). Дополнительно были собраны данные о структуре связей учащихся до поступления ("Укажите, с кем Вы были знакомы до поступления»). Число номинаций в сетевых вопросах было не ограничено. Информация об академической успеваемости учащихся была выгружена из административной базы данных. Также были использованы данные о поле учащихся и об их учебной группе. Коэффициент Жаккара за все периоды составлял порядка 0,35 - это свидетельствует, что в сети происходили необходимые для успешного моделирования объемы изменений. Потерянные данные составили $10 \%$, и авторы не использовали методы восполнения. В соответствии 
с рекомендациями разработчиков [Ripley et al., 2020], $10 \%$ потерянных данных не оказывают систематического влияния на моделирование, в свою очередь, большие потери данных необходимо специализированно восполнять.

Для каждой из сетей (дружба и помощь) были протестированы гипотезы о наличии социальной селекции и социального влияния. Как отмечалось выше, процессы в сетях могут протекать одновременно. В данной статье мы рассмотрим, каким образом происходила коэволюция связей и успеваемости в сети дружбы. Результаты моделирования приведены в таблице 2.

Таблица 2. Эффекты коэволюции сети дружбы и академической успеваемости

\begin{tabular}{|c|c|}
\hline Параметр & Коэффициент (Ст. ошибка) \\
\hline Параметр изменений 1 & $16,996(1,502) * * *$ \\
\hline Параметр изменений 2 & $15,311(1,577) * * *$ \\
\hline \multicolumn{2}{|l|}{ Контрольные сетевые эффекты } \\
\hline Плотность & $-2,260(0,120) * * *$ \\
\hline Взаимность & $1,690(0.107) * * *$ \\
\hline Популярность & $-0,029(0,011) *$ \\
\hline Активность & $0,026(0,010) * *$ \\
\hline \multicolumn{2}{|l|}{ Триадные эффекты } \\
\hline Транзитивность & $0,330(0,028) * * *$ \\
\hline 3-циклы & $-0,175(0,060) * *$ \\
\hline Взаимные триады & $-0.120(0,054) *$ \\
\hline Посредничество & $-0,131(0,028) * * *$ \\
\hline \multicolumn{2}{|l|}{ Связи в другой сети } \\
\hline Знакомство до поступления & $0,939(0,132) * * *$ \\
\hline Обучение в одной группе & $0,687(0,073) * * *$ \\
\hline Связь в сети помощи & $0,043(0,011) * * *$ \\
\hline \multicolumn{2}{|l|}{ Гендерные эффекты } \\
\hline Пол альтера (1-мужчина) & $0,110(0,065)$ \\
\hline Пол эго (1 - мужчина) & $0,161(0,063) *$ \\
\hline Схожесть по полу (социальная селекция) & $0,240(0,058) * * *$ \\
\hline \multicolumn{2}{|l|}{ Эффекты успеваемости } \\
\hline Успеваемость альтера (1 - мужчина) & $0,132(0,057) *$ \\
\hline Успеваемость эго (1 - мужчина) & $0,219(0,053) * * *$ \\
\hline Схожесть по успеваемости & $0,412(0,219)$ \\
\hline \multicolumn{2}{|l|}{ Динамика поведения } \\
\hline Параметр изменений 1 & $0,550(0,111) * * *$ \\
\hline Параметр изменений 2 & $1,132(0,251) * * *$ \\
\hline Линейный эффект & $1,134(0,629)$ \\
\hline Квадратный эффект & $0,452(0,447)$ \\
\hline Схожесть по полу (социальное влияние) & $7,797(3,890) *$ \\
\hline Влияние популярности на успеваемость & $0,041(0,099)$ \\
\hline Влияние активности на успеваемость & $-0,129(0,126)$ \\
\hline
\end{tabular}

Примечание. Статистическая значимость: *** $-p<0,001, * *-p<0,01, *-p<0,05$. 
Значимые эффекты параметров изменений [1, 2, 20 и 21] свидетельствуют о том, что в сети зафиксированы процессы изменений. Большее значение параметров изменений для первого периода по сравнению со вторым свидетельствует о том, что во второй период происходит меньше изменений.

Контрольные сетевые эффекты [3-10] статистически значимы. Отрицательный эффект плотности показывает, что акторы не склонны формировать связи случайным образом и образуют отношения, уже вовлеченные в сложные конфигурации. Положительный эффект взаимности говорит о том, что студенты склонны дружить с теми, кто уже проявляет дружеское отношение к ним. Комбинация положительной транзитивности и отрицательных 3-циклов свидетельствует о присутствии локальной иерархии. Отрицательный эффект взаимных триад [Block, 2015] демонстрирует, что транзитивность играет меньшую роль, нежели взаимность. Комбинация положительной транзитивности и негативного эффекта посредничества показывают, что студенты не ищут позиции брокерства. Положительный эффект активности свидетельствует о том, что с течением времени активные студенты формируют больше связей. Отрицательное значение популярности демонстрирует, что студенты не склонны образовывать связи дружбы с учащимися, уже вовлеченными во множественные связи.

Положительные и статистически значимые эффекты связи в других сетях [1113] свидетельствуют о том, что дружба возникает с большей вероятностью между одногруппниками, студентами, знакомыми до поступления, а также учащимися, с которыми они уже связаны в сети помощи.

Гендерные эффекты [14-16] демонстрируют, что мужчины более активны в создании связей [15]. Положительный эффект социальной селекции на основе пола [16] показывает, что студенты склонны формировать связи дружбы с представителями своего пола.

Эффекты успеваемости [17-19] демонстрируют, что высоко успевающие студенты как сами больше номинируют [18], так и получают больше номинаций [17]. В то же время эффект социальной селекции на основе академических достижений [19] статистически не значим. То есть нельзя сказать, что студенты склонны выбирать друзей со схожими академическими результатами.

Из эффектов поведения [22-26] статистическая значимость зафиксирована у социального влияния [24]. Этот эффект свидетельствует о том, что студенты адаптируют академическую успеваемость своих друзей.

Авторами был проведен тест на гетерогенность эффектов во времени [Ripley et al., 2020]. Этот тест традиционно используется в САОМ для оценки того, насколько значения коэффициентов целевой функции устойчивы в различные периоды. Тест было необходимо провести, так как данные были собраны трижды, и изменения между $t_{1} \rightarrow t_{2}$ и $t_{2} \rightarrow t_{3}$ могли быть обусловлены разными процессами. Была зафиксирована гетерогенность в таких параметрах, как знакомство до поступления, а также в эффектах успеваемости. Часть этих эффектов легко объяснима - в частности, очевидно, что знакомство до поступления в вуз важно на начальных этапах создания сети, и его роль нивелируется по мере обучения.

В итоге авторы построили модель для выявления ключевых движущих сил эволюции сети дружбы и академических достижений студентов. Они продемонстриро- 
вали, что в социальной сети дружбы акторы склонны адаптировать академические достижения своих друзей. Таким образом, сделан вывод о высокой роли связей дружбы в процессе передачи знаний.

\section{Ограничения метода}

САОМ призваны проанализировать поведение участников небольшой социальной общности, в которой акторы осведомлены о связях и академических результатах друг друга [Snijders, Van de Bunt, Steglich, 2010]. При этом результаты, полученные для данной конкретной группы, не могут быть перенесены на более широкие выборки и социальные контексты. Такая ситуация характерна для всех работ, предполагающих подробное исследование одной социальной сети (complete network design) [Hanneman, Riddle, 2005]. Дополнительно, несмотря на уникальные достоинства САОМ и возможности разделения процессов социальной селекции и влияния, эти модели не позволяют оценить размер эффектов, ограничивая возможности в интерпретации результатов исследования и сопоставлении результатов.

\section{Перспективы исследований}

Число работ с использованием сетевого инструментария, в том числе и САОМ, растет год от года. Анализ социальных сетей предлагает сместить фокус с анализа взаимосвязи между переменными на структуру и динамику группы в целом, что ставит много новых исследовательских вопросов.

K настоящему моменту большинство публикаций, использующих в качестве метода исследования САОМ, реализованы на опросных данных об учащихся [Henneberger, Mushonga, Preston, 2021; Vörös et al., 2020]. Это свидетельствует о перспективах изучения структуры и динамики социальных сетей в группах респондентов более зрелого возраста, а также в группах с участниками разного возраста. В большинстве своем исследователи собирают данных о положительных взаимодействиях (дружба, помощь) [Lomi et al., 2011; Palacios, Berger, 2015], хотя все чаще предпринимаются попытки анализа негативных связей [Boda, Néray, Snijders, 2020]. Существующие работы крайне редко изучают иные типы социальных взаимодействий, в частности онлайн-коммуникацию. Предполагаем, что число публикаций, рассматривающих эволюцию онлайн-сетей и влияние онлайн-связей на индивидуальные результаты и благополучие, в ближайшие годы вырастет в связи с беспрецедентным влиянием онлайн-коммуникации на социальную жизнь в период пандемии COVID-19.

Важно отметить, что существующие работы с использованием CAOM ограничены в кросс-культурных контекстах [Henneberger, Mushonga, Preston, 2021]. Подавляющее большинство исследований реализовано на данных об учащихся из западных демократических обществ. Несмотря на то, что такие выборки репрезентируют поведение $12 \%$ популяции всего населения Земли, на них проводится 80 \% работ [Henrich, Heine, Norenzayan, 2010]. В то же время исследования фиксируют значительную кросс-культурную вариацию в процессах социализации (см., например, [Henneberger, Mushonga, Preston, 2021]), что свидетельствует о необходимости изучения разнообразных выборок. 


\section{Список литературы (References)}

Валеева Д.Р., Польдин О.В., Юдкевич М. М. Связи дружбы и помощи при обучении в университете // Вопросы образования. 2013. № 4. С. 70-84. https:// doi.org/10.17323/1814-9545-2013-4-70-84 (дата обращения 21.03.2021).

Valeeva D. R., Poldin O. V., Yudkevich M. M. (2013) Friendly Relationships and Relationships of Assistance at a University. Educational Studies. Vol. 4. P. 70-84. https:// doi.org/10.17323/1814-9545-2013-4-70-84. (In Russ.)

Докука С. В., Валеева Д.Р. Статистические модели для анализа динамики социальных сетей в исследованиях образования // Вопросы образования. 2015. № 1. C. 201-213. https://doi.org/ 10.17323/1814-9545-2015-1-201-213.

Dokuka S. V., Valeeva D. R. (2015) Statistical Models for Analysis of Social Network Dynamics in Educational Studies. Educational Studies. Vol. 1. P. 201-213. https:// doi.org/ 10.17323/1814-9545-2015-1-201-213. (In Russ.)

Харари Ф. Теория графов. М.: Мир, 1973.

Harary F. (1973) Graph Theory. Moscow: Mir. (In Russ.)

Block P. (2015) Reciprocity, Transitivity, and the Mysterious Three-Cycle. Social Networks. Vol. 40. P. 163-173. https://doi.org/10.1016/j.socnet.2014.10.005.

Block P., Grund T. (2014) Multidimensional Homophily in Friendship Networks. Network Science. Vol. 2. No. 2. P. 189-212. https://doi.org/10.1017/nws.2014.17.

Boda Z., Néray B., Snijders T. A. (2020) The Dynamics of Interethnic Friendships and Negative Ties in Secondary School: The Role of Peer-Perceived Ethnicity. Social Psychology Quarterly. Vol. 8. No. 4. P. 342-362. https://doi.org/10.1177/0190272520907594.

Brouwer J., Flache A., Jansen E., Hofman A., Steglich C. (2018) Emergent Achievement Segregation in Freshmen Learning Community Networks. Higher Education. Vol. 76. No. 3. P. 483-500. https://doi.org/10.1007/s10734-017-0221-2.

de la Haye K., Embree J., Punkay M., Espelage D. L., Tucker J. S., Green Jr., H. D. (2017) Analytic Strategies for Longitudinal Networks With Missing Data. Social Networks. Vol. 50. P. 17-25. https://doi.org/10.1016/j.socnet.2017.02.001.

DeLay D., Zhang L., Hanish L.D., Miller C. F., Fabes R. A., Martin C. L., Kochel K. P., Updegraff K. A. (2016) Peer Influence on Academic Performance: A Social Network Analysis of Social-Emotional Intervention Effects. Prevention Science. Vol. 17. No. 8. P. 903-913. https://doi.org/10.1007/s11121-016-0678-8.

Dokuka S., Valeeva D., Yudkevich M. (2020) How Academic Achievement Spreads: The Role of Distinct Social Networks in Academic Performance Diffusion. Plos ONE. Vol. 15. No. 7. e0236737. https://doi.org/10.1371/journal.pone.0236737.

Grunspan D. Z., Eddy S. L., Brownell S. E., Wiggins B. L., Crowe A. J., Goodreau S. M. (2016) Males Under-Estimate Academic Performance of Their Female Peers in Undergraduate Biology Classrooms. PloS ONE. Vol. 11. No. 2. e0148405. https:// doi.org/10.1371/journal.pone.0148405. 
Ellwardt L., Steglich C., Wittek R. (2012) The Co-Evolution of Gossip and Friendship in Workplace Social Networks. Social Networks. Vol. 34. No. 4. P. 623-633. https:// doi.org/10.1016/j.socnet.2012.07.002.

Flashman J. (2012) Academic Achievement and Its Impact on Friend Dynamics. Sociology of Education. Vol. 85. No. 1. P. 61-80. https://doi.org/10.1177/0038040711417014. Hanneman R. A., Riddle M. (2005) Introduction to Social Network Methods. Riverside: University of California.

Henneberger A. K., Mushonga D. R., Preston A. M. (2021) Peer Influence and Adolescent Substance Use: A Systematic Review of Dynamic Social Network Research. Adolescent Research Review. Vol. 6. No. 1. P. 57-73. https://doi.org/10.1007/s40894-01900130-0.

Henrich J., Heine S. J., Norenzayan A. (2010) Most People Are Not WEIRD. Nature. Vol. 466. No. 7302. https://doi.org/10.1038/466029a.

Jaccard P. (1900) Contribution au problème de l'immigration post-glaciare de la flore alpine. Bulletin de la Société Vaudoise des Sciences Naturelles. Vol. 36. No. 136. P. 87 130. https://www.e-periodica.ch/digbib/view?pid=bsv-002:1900:36::854\#141.

Kretschmer D., Leszczensky L., Pink S. (2018) Selection and Influence Processes in Academic Achievement - More Pronounced for Girls? Social Networks. Vol. 52. P. 251-260. https://doi.org/10.1016/j.socnet.2017.09.003.

Lomi A., Snijders T. A., Steglich C. E., Torló V. J. (2011) Why Are Some More Peer Than Others? Evidence From a Longitudinal Study of Social Networks and Individual Academic Performance. Social Science Research. Vol. 40. No. 6. P. 1506-1520. https:// doi.org/10.1016/j.ssresearch.2011.06.010.

Palacios D., Berger C. (2015) Are Good Students Desirable Friends? Evidence for Friendship Selection Among Elementary Students/¿Son los buenos estudiantes amigos deseables? Evidencia para la selección de amistad entre estudiantes de educación primaria. Estudios de Psicología. Vol. 36. No. 2. P. 496-508. https://doi.org/10.1080/ 02109395.2015.1028729.

Palacios D., Dijkstra J. K., Villalobos C., Treviño E., Berger C., Huisman M., Veenstra R. (2019) Classroom Ability Composition and the Role of Academic Performance and School Misconduct in the Formation of Academic and Friendship Networks. Journal of School Psychology. Vol. 74. P. 58-73. https://doi.org/10.1016/j.jsp.2019.05.006.

Prell C. (2012) Social Network Analysis: History, Theory and Methodology. London: Sage.

Rambaran J. A., Hopmeyer A., Schwartz D., Steglich C., Badaly D., Veenstra R. (2017) Academic Functioning and Peer Influences: A Short-Term Longitudinal Study of Network - Behavior Dynamics in Middle Adolescence. Child development. Vol. 88. No. 2. P. 523-543. https://doi.org/10.1111/cdev.12611.

Ripley R. M., Snijders T. A. B., Bóda Z., Vörös A., Preciado P. (2020) Manual for RSiena. Oxford: University of Oxford, Department of Statistics; Nuffield College. 
Robins G. (2013) A Tutorial on Methods for the Modeling and Analysis of Social Network Data. Journal of Mathematical Psychology. Vol. 57. No. 6. P. 261-274. https:// doi.org/10.1016/j.jmp.2013.02.001.

Robins, G., Pattison, P., Kalish, Y., Lusher, D. (2007) An Introduction to Exponential Random Graph ( $\left.P^{*}\right)$ Models for Social Networks. Social Networks. Vol. 29. No. 2. P. 173-191. URL: https://doi.org/10.1016/j.socnet.2006.08.002 (accessed: 20.03.2021).

Snijders T. A., Van de Bunt G. G., Steglich C. E. (2010) Introduction to Stochastic ActorBased Models for Network Dynamics. Social Networks. Vol. 32. No. 1. P. 44-60. URL: https://doi.org/10.1016/j.socnet.2009.02.004 (accessed: 20.03.2021).

Vörös A., Boda Z., Elmer T., Hoffman M., Mepham K., Raabe I.J., Stadtfeld C. (2020) The Swiss StudentLife Study: Investigating the Emergence of an Undergraduate Community Through Dynamic, Multidimensional Social Network Data. Social Networks. Vol. 65. P. 71-84. https://doi.org/10.1016/j.socnet.2020.11.006.

Wasserman S., Faust K. L.M. (1994) Social Network Analysis: Methods and Applications. Cambridge: Cambridge University Press.

Zandberg T., Huisman M. (2019) Missing Behavior Data in Longitudinal Network Studies: The Impact of Treatment Methods on Estimated Effect Parameters in Stochastic Actor Oriented Models. Social Network Analysis and Mining. Vol. 9. No. 1. https://doi.org/ 10.1007/s13278-019-0553-2. 